\title{
Comprehensive protection of data-partitioned video for broadband wireless IPTV streaming
}

\author{
Laith Al-Jobouri, Martin Fleury* and Mohammed Ghanbari \\ Department of CSEE, University of Essex, Essex, UK
}

\begin{abstract}
This paper examines the threat to video streaming from slow and fast fading, traffic congestion, and channel packet drops. The proposed response is a combination of: rateless channel coding, which is adaptively applied; data-partitioned source coding to exploit prioritized packetization; and duplicate slice provision, which is the focus of the evaluation in this paper. The paper also considers the distribution of intra-refresh macroblocks as a means of avoiding sudden data rate increases. When error bursts occur, this paper shows that duplicate slices are certainly necessary but this provision is more effective for medium quality video than it is for high quality video. The percentage of intra-refresh macroblocks can be low and still reduce the impact of temporal error propagation.
\end{abstract}

Keywords: Data-partitioning, error resilience, FEC, IPTV, rateless coding, redundant packets, video streaming, WiMAX

\section{Introduction}

Broadband wireless delivery of video streaming for IPTV is under active investigation, though this environment is challenging due to signal noise and interference, leading to error bursts. In the time division duplex (TDD) form of multiplexing favored by broadband wireless access networks such as IEEE 802.16e (mobile WiMAX) [10], Automatic Repeat reQuests (ARQs) come almost for free by virtue of the return sub-frame. Therefore, a form of type-1 hybrid ARQ is possible. To achieve this, the paper introduces an adaptive variant of rateless channel coding which utilizes such retransmissions. To avoid the long start-up delays that can arise from packet erasure correction, error correction in the proposed protection scheme is at the byte level. However, byte-level channel coding is powerless against packet drops, as obviously a packet can be lost through buffer overflow before forward error correction (FEC) can be applied. Outright packet drops on the channel can also occur, for example if the signal strength fails to reach a desired receiver threshold. Therefore, further protection is required, which in this paper is provided by duplicate video packets. The paper tests the effectiveness of different levels of duplication according to the source coding scheme used.

We have also employed video data-partitioning [19] which is a form of error-resilient source coding in which the compressed video data are reorganized according to decoding priority. In an H.264/AVC (Advanced Video Coding) codec [35], the data are partitioned between packets rather than re-ordered within a packet (refer to Section 2 for details). In particular, motion vectors (MVs) are packed into a partition-A slice packet, allowing error concealment to reconstruct missing partition-C slices containing

\footnotetext{
${ }^{*}$ Corresponding author: Martin Fleury, Department of CSEE, University of Essex, Wivenhoe Park, Colchester CO4 3SQ, Essex, UK. Tel.: +44 1206 872684; Fax: +44 1206 872900; E-mail: fleum@essex.ac.uk.
} 
texture data (quantized transform coefficient residuals). One advantage of this arrangement is that, as partition-A packets can be relatively smaller than the other packets (according to the compression ratio) then these packets are less likely to be corrupted by channel noise or dropped at buffers.

It is also possible to use redundant picture slices [27] rather than duplicate slices. Redundant picture slices employ a higher quantization parameter $(\mathrm{QP})$ and, hence, coarser quantization than the original slices. However, to use redundant picture slices would cause additional drift between the encoder and the decoder, if (say) a partition-A packet was matched with a partition-C packet's data with a different QP. Besides, there is an implementation issue. Though both redundant slices and data-partitioned slices co-exist in the Extended profile, they are not jointly implemented in the JM implementation of H.264/AVC [31] and, in fact, appear not to be implemented at all in most other software codec implementations such as QuickTime, Nero, and LEAD randomly to name a few. However, it is possible with data-partitioning through repeated runs of the encoder to create a duplicate stream of all partitionA slice packets or a duplicate stream consisting of partition-A and partition-B packets or, indeed, a replica of the original stream. Unequal error protection (UEP) is also associated with data partitioning, for example by means of hierarchical modulation in [4]. Nevertheless, the combined congestion and channel error protection in the proposed scheme negates the need for UEP and Equal Error Protection (EEP) is employed instead.

The feasibility of video streaming for broadband access has been established [11] by means of a live WiMAX testbed. However, that study [11] concentrated on varying the configuration parameters and used UDP-transported streams, seemingly without congestion or error control. Other work in [18] was primarily concerned with combining true- and near-video-on-demand, providing a solution to how content should be allocated between the two services. In [9], adaptive multicast streaming was proposed using the Scalable Video Coding (SVC) extension for H.264. Fixed WiMAX channel conditions were monitored in order to vary the bitrate accordingly. Unfortunately, the subsequent decision of the JVT standardization body for H.264/AVC not to support fine-grained scalability (FGS) implies that it will be harder to respond to channel volatility. Other work has also investigated combining scalable video, with multi-connections in [14] and in comparison with H.264/AVC in [5]. However, the data-dependencies between layers in H.264/SVC medium grained scalability are a concern, as, unlike in FGS, enhancement layer packets may successfully arrive but be unable to be reconstructed if key pictures also fail to arrive. Instead, data-partitioning in this paper can be viewed as a simplified form of SNR or quality layering [24] which can be protected against congestion drops through duplicate slices. Temporal scalability is avoided because of its impact on media synchronization, especially lip synchronization.

There remains the problem of channel errors, which in this paper are guarded against in two ways. Firstly, to reduce temporal error propagation in H.264/AVC it is possible to place intra-refresh macroblocks (MBs) amongst the normally inter-coded MBs of P-pictures. These MBs are placed with naturally encoded inter-coded MBs within partition-B slices. We have investigated the correct way to provision intra-refresh MBs. Because such intra-coded MBs are not as efficient to code as when predictive inter-coding is applied, the maximum level of intra-refresh MBs should be assessed to avoid excess overhead.

Secondly, a more important form of protection is the use of application-layer FEC, which in this paper is through rateless channel coding [23]. Raptor coding [29] in particular is a systematic variety of rateless code that does not share the high error floors [25] of prior rateless codes. It also has $\mathrm{O}(\mathrm{n})$ decoding computational complexity. A point to note is that rateless codes are a probabilistic channel code in the sense that reconstruction is not guaranteed. Because Raptor coding is indeed rateless, it is possible to adaptively vary the amount of redundant data according to an estimate of the channel conditions. It is 
Table 1

NAL unit types

\begin{tabular}{ccl}
\hline NAL unit type & Class & Content of NAL unit \\
\hline 0 & - & Unspecified \\
1 & VCL & Coded slice \\
2 & VCL & Coded slice partition A \\
3 & VCL & Coded slice partition B \\
4 & VCL & Coded slice partition C \\
5 & VCL & Coded slice of an IDR picture \\
$6-12$ & Non-VCL & Suppl. info., Parameter sets, etc. \\
$13-23$ & - & Reserved \\
$24-31$ & - & Unspecified \\
\hline
\end{tabular}

also possible to piggyback additionally generated redundant data if the estimate is insufficient to allow reconstruction of the packet or the belief propagation decoding algorithm fails to run to completion. However, further details are reserved for Section 4.

We have investigated video streaming in the context of IPTV. IPTV services include live TV programon-demand, which can be 'start-again' TV, as well as 'catch-up' TV [7], both of which require unicast streaming. Video-on-demand can also be provided as a part of an IPTV service. However, if this form of IPTV is to be extended to mobile devices then wireless access [36] presents a challenging environment for video streaming, especially if sport scenes are streamed, as these often contain rapid motion, leading to high temporal coding complexity. Error bursts can disrupt the fragile compressed bitstream (because of predictive coding dependencies), while error control should not increase end-to-end latency because of display and decode deadlines. However, as intelligent content management of IPTV moves popular material nearer to the end-user [34], it becomes feasible to combine application-layer channel coding with retransmission of additional redundant data without incurring prohibitive delays to real-time video display.

The remainder of this paper is organized as follows. Section 2 discusses the two forms of error resilience source coding employed in the proposed protection scheme. The following Section briefly introduces rateless channel coding. Section 4 discusses the protection schemes active features: channel coding adaptation, retransmission requests, and slice duplication. Section 5 describes the simulation configuration used to evaluate the scheme. Section 6 provides results according to duplicate packet and intra-refresh provision. Finally, Section 7 draws some conclusions.

\section{Error resilience measures}

This Section describes the two source coding error resilience measures used to protect the data before channel coding.

\section{Data-partitioning}

The H.264/AVC codec conceptually separates the Video Coding Layer (VCL) from the Network Abstraction Layer (NAL). The VCL specifies the core compression features, while the NAL supports delivery over various types of network. In a communication channel, the quality-of-service is affected by the two parameters of bandwidth and the probability of error. Therefore, as well as video compression efficiency, which is provided for through the VCL layer, adaptation to communication channels should be 
carefully considered. The concept of the NAL, together with the error-resilience features in H.264/AVC, allows communication over a variety of different channels. Table 1 is a summarized list of different NAL unit types. NAL units 1 to 5 contain different VCL data that will be described later. NAL units 6 to 12 are non-VCL units containing additional information such as parameter sets and supplemental information.

Each frame in H.264/AVC can be divided into several slices; each of which contains a flexible number of MBs. Variable Length Coding (VLC) that is entropy coding of the compressed data takes place as the final stage of the hybrid codec. In H.264/AVC, arithmetic coding replaced other forms of entropy coding in earlier codecs. In each slice, the arithmetic coder is aligned and its predictions are reset. Hence, every slice in the frame is independently decodable. Therefore, slices can be considered as points of decoder resynchronization that prevent error propagation to the entire picture. Each slice is placed within a separate NAL unit (see Table 1). The slices of an Instantaneous Decoder Refresh- (IDR-) ${ }^{1}$ or I-picture (i.e. a picture with all intra slices) are located in type 5 NAL units, while those belonging to a non-IDR or I-picture (P- or B-pictures) are placed in NAL units of type 1, and in types 2 to 4 when data-partitioning mode is active, as now explained.

In type 1 and type 5 NALs, MB addresses, MVs and the transform coefficients of the blocks, are packed into the packet, in the order they are generated by the encoder. In Type 5, all parts of the compressed bitstream are equally important, while in type 1, the MB addresses and MVs are much more important than the (integer-valued) Discrete Cosine Transform (DCT) coefficients. In the event of errors in that type of packet, the fact that symbols appearing earlier in the bit-stream suffer less from errors than those which come later ${ }^{2}$ means that bringing the more important parts of the video data (such as headers and MVs) ahead of the less important data or separating the more important data altogether for better protection against errors can significantly reduce channel errors. In the standard video codecs, this practice is known as data partitioning.

However, in H.264/AVC when data partitioning is enabled, every slice is divided into three separate partitions and each partition is located in either of type- 2 to type-4 NAL units, as listed in Table 1. A NAL unit of type 2, also known as partition A, comprises the most important information of the compressed video bit stream of P- and B-pictures, including the MB addresses, MVs, and essential headers. If any MBs in these pictures are intra-coded, their DCT coefficients are packed into the type-3 NAL unit, also known as partition-B. Type 4 NAL, also known as partition-C, carries the DCT coefficients of the motion-compensated inter-picture coded MBs.

In the context of H.264/AVC data-partitioning, it should be pointed out that though partition-A is independent of partitions B and C, constrained intra prediction should be set [24] to make partition$B$ independent of partition- $C$. However, partition- $C$ cannot be made independent of partition- $B$ if the Context-Adaptive Variable Length Coding (CAVLC) option is set. As data-partitioning is currently only enabled in the H.264/AVC Extended profile, whereas the alternative Context Adaptive Binary Arithmetic Coding (CABAC) option is not available in the Extended profile, it seems that partition-C is inevitably dependent on partition-B.

\subsection{Intra-refresh macroblocks}

In the proposed robust scheme, an issue is how to protect against the temporal error propagation that

\footnotetext{
${ }^{1}$ An IDR picture is confusedly equivalent to an I-picture in previous standards. An I-picture in H.264/AVC allows predictive references beyond the boundary of a GOP.

${ }^{2}$ Because of the cumulative effect of VLC, symbols nearer the slice synchronization marker suffer less from errors than those that appear later in a bitstream.
} 
can occur whenever predictively-coded P-frames are lost. A traditional way to do this is to insert periodic, intra-coded I-frames, usually every 12 or 15 frames that is every half-second according to frame rate. The spatially-encoded macroblocks (MBs) of the I-frame halt any temporal error propagation and act as anchor points for a future set of frames. Unfortunately, the insertion of I-frames leads to sudden data transmission increases due to the coding inefficiency of spatial referencing. It can also lead to buffering delays as the multiple packets associated with I-frames are formed and transmitted. Therefore, distributed insertion of intra-refresh MBs is an alternative. In the JM codec implementation of the H/264/AVC standard, two main methods of distributed insertion are available: either random placement of IR MBs within each frame; or placing a line of intra-refresh MBs within each P-frame on a cyclic basis.

In the latter forced intra-refresh method, the line size can be increased to a region or slice [32] in order to control the rate that the total picture area is refreshed. Against this suggestion must be balanced the overhead from including a complete line or region of MBs, as such MBs are costly to encode. In fact, in this paper it is suggested that despite the apparent advantages of the cyclic line method, at least in respect to data-partitioned video compression, random selection of intra-refresh MBs is preferable. Random intra refresh may on occasion select existing intra-coded MBs but it has the advantage that in the JM implementation of H.264/AVC the overhead from intra-coded MBs is readily controllable. This is because intra-refresh MBs are not the only form of intra-coded MBs, as encoders will insert such MBs when new areas of a picture are revealed, as may especially happen when there is rapid motion within a sequence. Thus, the forced intra-refresh method does not account for areas of the region that may already have been intra-coded. It also has another weakness in that future motion prediction may occur from regions of a prior picture yet to be refreshed. This defect can be remedied, possibly by restricting the range of the MVs within the refreshed region [17] or by observing the direction of motion within a sequence [28]. However, these alternatives [17,28] add coding complexity to the intra-refresh process and may be unnecessary when processing a low-motion video sequence. Another possibility is to adaptively alter the extent of MB provision [21] according to scene content and channel conditions. This is most suited to live encoding and is not a general method.

One advantage of forced intra-refresh is that it provides a natural channel-swapping (or zapping) point at the start of each refresh cycle, just as periodic I-frames provided. In catch-up TV systems, for example the BBC's iPlayer, such a facility is not required, as no channels are swapped. If for channel swapping, gradual decoder refresh is performed instead of periodic refresh then growing the refresh area from an isolated region is preferable to random $\mathrm{MB}$ refresh, as the subjective visual effect is better. This facility was proposed to the Joint Video team developing the H.264/AVC codec [21] but is not currently implemented, perhaps because a method of signaling a switching point is the subject of a patent.

A point to note is the different way that random intra-refresh MBs are specified in the H.264/AVC JM 14.2 implementation compared to that of cyclic IR line intra update. For random intra-refresh MBs, a maximum percentage of intra-refresh can be specified, which percentage includes existing naturally encoded intra-coded MB. If the given quota of intra-refresh MB is already largely occupied by naturallyencoded intra-coded MBs, then only a small amount of extra randomly inserted MBs will be added. In contrast, if a line of intra-refresh MBs is inserted then these MBs are added in addition to those intra-coded MBs that have already been included by the encoder.

Figure 1 is a comparison between the relative sizes of the partitions according to QP for the video clip which is employed in Section 6's evaluation. The test sequence was Football, which is a scene with rapid movement and consequently has a high temporal coding complexity. This sequence is of a content type that quality of experience subjective testing indicates currently provides a difficult viewing 


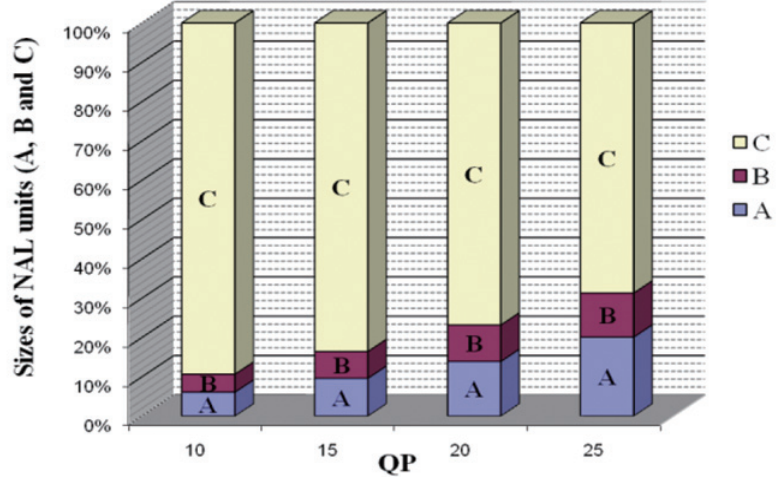

(a)

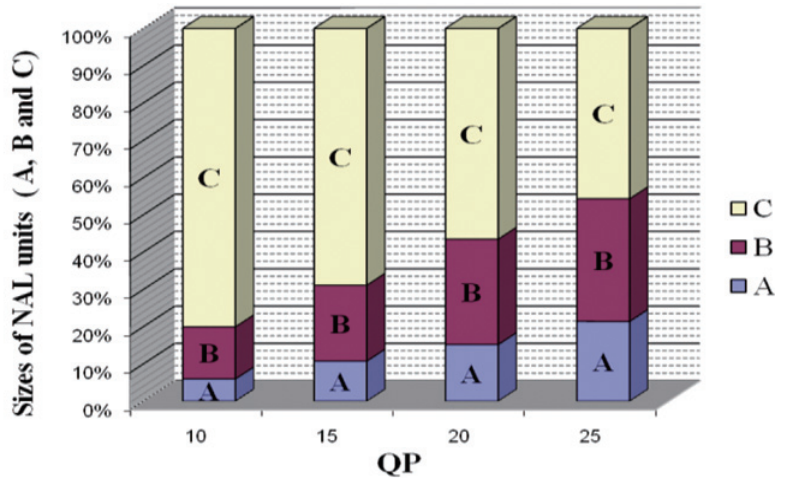

(b)

Fig. 1. Relative sizes of data partitions according to quantization parameter (QP) for the Football video sequence, with (a) $5 \%$ intra-coded refresh MBs, and (b) 25\% intra-coded refresh MBs.

experience [1] on mobile devices. Football was Variable Bit Rate (VBR) encoded with 4:2:0 chroma subsampling at Common Intermediate Format (CIF) $(352 \times 288$ pixels/picture), with a Group-of-Pictures (GOP) structure of IPPP. . . at $30 \mathrm{~Hz}$, i.e. one initial I-picture followed by all predictive P-pictures. This arrangement removes the complexity of bi-predictive B-pictures at a cost in an increased bit-rate. Notice that the range of QP in H.264/AVC is 0-51 with higher values corresponding to higher compression ratios and lower quality video. From the Figure it is apparent that the partition-B's contribution increases as the percentage of intra-refresh MBs grows, making partition-B packets more vulnerable to congestion and channel error as a result. The size of partition-C bearing packets declines with increasing QP as a result of coarser quantization of transform coefficients.

Table 2 is a more complete comparison of the actual sizes of the partitions' contributions according to QP for the Football sequence. From the Table, again it is apparent that, as the percentage of intrarefresh MBs is increased, the size in bytes of partition-B increases for the same QP. Because more MBs are assigned to partition- $\mathrm{B}$, the size of partition- $\mathrm{C}$ reduces. Because of the relatively large amount of naturally intra-encoded MBs, this effect is gradual until $25 \%$ of random intra-refresh MBs is added. A point to notice is that the total size of the stream declines significantly with coarser quantization due to a higher QP. Because intra-coding is less efficient, the total sizes increase as the percentage of intra-refresh MBs is increased, making the percentage of intra-refresh MBs a significant parameter to examine in any transmission scheme.

\section{Rateless channel coding}

We have used rateless channel coding, specifically Raptor coding, in the protection scheme. Rateless or Fountain coding [23], of which Raptor coding [29] is a subset, is ideally suited to a binary erasure channel in which either the lower-layer error-correcting code works or the channel decoder fails and reports that it has failed to the application layer. In erasure coding, flawed data symbols may be reconstructed from a set of successfully received symbols (if sufficient of these symbols are successfully received). The class of Fountain codes allows a continual stream of additional symbols to be generated in the event that the original symbols could not be decoded. It is the ability to easily generate new symbols that makes Fountain codes rateless. Decoding will succeed with small probability of decode failure if any of $k(1+$ 
Table 2

Size of different data partitions' contribution in byte, as a mean over Football's frames at various QP for various levels of intra-refresh MBs

\begin{tabular}{ccccc}
\hline QP & \multicolumn{4}{c}{ 2\% Intra-refresh MB } \\
\cline { 2 - 5 } & $\mathrm{A}$ & $\mathrm{B}$ & $\mathrm{C}$ & Total \\
\hline 20 & 1842 & 2678 & 3889 & 8409 \\
25 & 1687 & 1697 & 2533 & 5917 \\
30 & 1459 & 1047 & 1496 & 4002 \\
35 & 1117 & 572 & 688 & 2377 \\
\hline QP & \multicolumn{5}{c}{$5 \%$ Intra refresh MB } \\
\cline { 2 - 5 } & $\mathrm{A}$ & $\mathrm{B}$ & $\mathrm{C}$ & Total \\
\hline 20 & 1845 & 2767 & 3867 & 8479 \\
25 & 1690 & 1763 & 2511 & 5964 \\
30 & 1463 & 1082 & 1482 & 4027 \\
35 & 1120 & 595 & 682 & 2397 \\
\hline $\mathrm{CP}$ & \multicolumn{5}{c}{$6 \%$ Intra-refresh MB } \\
\cline { 2 - 5 } $\mathrm{A}$ & $\mathrm{B}$ & $\mathrm{C}$ & Total \\
\hline 20 & 1846 & 2810 & 3850 & 8506 \\
25 & 1696 & 1793 & 2502 & 5991 \\
30 & 1467 & 1098 & 1479 & 4044 \\
35 & 1123 & 604 & 681 & 2408 \\
\hline QP & \multicolumn{5}{c}{$25 \%$ Intra-refresh MB } \\
\cline { 2 - 5 } $\mathrm{A}$ & $\mathrm{B}$ & $\mathrm{C}$ & Total \\
\hline 20 & 1893 & 3450 & 3669 & 9012 \\
25 & 1746 & 2216 & 2379 & 6341 \\
30 & 1505 & 1346 & 1405 & 4256 \\
35 & 1146 & 729 & 646 & 2521 \\
\hline QP & \multicolumn{5}{c}{ MB Line Intra Update } \\
\cline { 2 - 5 } $\mathrm{A}$ & $\mathrm{B}$ & $\mathrm{C}$ & Total \\
\hline 20 & 1885 & 3385 & 3683 & 8953 \\
25 & 3683 & 2160 & 2400 & 8243 \\
30 & 1498 & 1312 & 1414 & 4224 \\
35 & 1143 & 716 & 652 & 2511 \\
\hline \multicolumn{5}{c}{}
\end{tabular}

$\varepsilon$ ) symbols are successfully received, where $k$ is the number of source symbols and $\varepsilon$ is a low percentage of coding overhead. For example, in [2] for rateless coding of Internet video streams that overhead was $5 \%$. In its simplest form, the symbols are combined in an exclusive OR (XOR) operation according to the order specified by a randomized, low-density generator matrix and, in this case, the probability of decoder failure is $\partial=2^{-k \varepsilon}$, which for large $k$ approaches the Shannon limit. The random sequence must be known to the receiver but this is easily achieved through knowledge of the sequence seed.

In general, encoding of rateless codes is accomplished as follows: Choose $d_{i}$ randomly from some distribution of degrees, where $\rho_{d i}=\operatorname{Pr}$ [degree $\left.d_{i}\right] ; \operatorname{Pr}$ is the probability of a given event. Choose $d_{i}$ random information symbols $R_{i}$ among the $k$ information symbols. These $R_{i}$ symbols are then XORed together to produce a new composite symbol, which forms one symbol of the transmitted packet. Thus, if the symbols are bytes then all of the $R_{i}$ byte's bits are XORed with all of the bits of the other randomly selected bytes in turn. It is not necessary to specify the random degree or the random symbols chosen if it is assumed that the (pseudo-)random number generators of sender and receiver are synchronized.

Symbols are processed at the decoder as follows. If a symbol arrives with degree greater than one it is buffered. If a clean symbol arrives with degree one then it is XORed with all symbols in which it was 
used in the encoding process. This reduces the degree of each of the symbols to which the degree-one symbol is applied. When a symbol is eventually reduced to degree one, it too can be used in the decoding process. Notice that a degree-one symbol is a symbol for which no XORing has taken place. Notice also that for packet erasure channels a clean degree-one symbol (a packet) is easily established as such. For byte-erasures the physical layer FEC can be reasonable expected to isolate clean symbols or blocks of clean symbols.

In the decoding process, the robust Soliton distribution [23] is employed as the degree distribution, as this produces degree-one symbols at a convenient rate for decoding. It also avoids isolated symbols that are not used elsewhere. Two tuneable parameters $c$ and $\delta$ serve to form the expected number of useable degree one symbols. Set

$$
S=c \ln \left(\frac{k}{\delta}\right) \sqrt{k}
$$

where $c$ is a constant close to 1 and $\delta$ is a bound on the probability that decoding fails to complete. Now define

$$
\begin{aligned}
\tau(d) & =\frac{S}{k} \frac{1}{d} \text { for } d=1,2, \ldots(k / S)-1 \\
& =\frac{S}{k} \ln \left(\frac{S}{\delta}\right) \text { for } d=k / S \\
& =0 \text { for } d>k / S
\end{aligned}
$$

as an auxiliary positive-valued function to give the robust Soliton distribution:

$$
\mu(d)=\frac{\rho(d)+\tau(d)}{z}
$$

where $z$ normalizes the probability distribution to unity and is given by:

$$
z=\sum_{d}(\rho(d)+\tau(d))
$$

Then, in order to model Raptor coding, we employed the following statistical model [22]:

$$
\begin{aligned}
P_{f}(m, k) & =1 & & \text { if } m<k, \\
& =0.85 \times 0.567^{m-k} & & \text { if } m \geqslant k,
\end{aligned}
$$

where $P_{f}(m, k)$ is the failure probability of the code with $k$ source symbols if $m$ symbols have been received. Notice that the authors of [22] remark and show that for $k>200$ the model almost perfectly models the performance of the code. In the protection scheme, the symbol size was set to bytes within a packet. Clearly, if instead 200 packets are accumulated before the rateless decoder can be applied (or at least Eq. (5) is relevant) there is a penalty in start-up delay for the video stream and a cost in providing sufficient buffering at the mobile stations.

\section{Protection scheme}

This Section establishes the protection scheme in the context of WiMAX wireless access. 


\begin{tabular}{|c|c|c|}
$\begin{array}{c}\text { MAC } \\
\text { header }\end{array}$ & Payload & CRC \\
$\underset{6 \text { bytes }}{\longrightarrow}$
\end{tabular}

Fig. 2. General format of a MAC PDU with optional CRC.

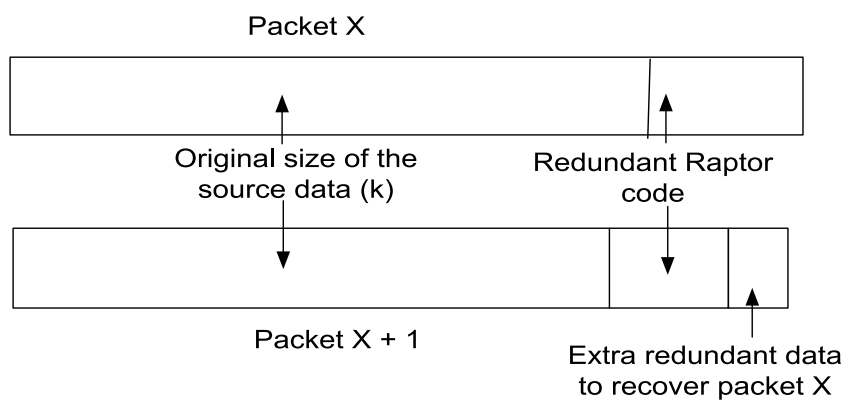

Fig. 3. Division of payload data in a packet (MPDU) between source data, original redundant data and piggybacked data for a previous erroneous packet.

\subsection{Packetization}

To try to ensure corrupted packets are reconstructed if the original estimated redundant data is insufficient for reconstruction, the IPTV scheme works by retransmitting piggybacked redundant data. To reduce latency, the number of such retransmissions, after an ARQ over the uplink, was limited to one. The application-layer receives only clean symbols (bytes) from any corrupted packets. This latter function is performed by PHYsical-layer FEC which passes up correctly received blocks of data (through a Cyclic Redundancy Check (CRC)) but suppresses erroneous data. Thus, in IEEE 802.16e [3], a binary, non-recursive convolutional encoder with a constraint length of 7 and a native coding rate of $1 / 2$ operates at the physical layer. Upon receipt of the correctly received data, decoding of the information symbols is attempted, which will fail with a probability given by Eq. (5) for $k>200$.

Figure 2 shows the general format of a WiMAX packet, with an optional CRC that could be used to check successful reconstruction or not of the packet after application of the rateless code (assuming cross-layer access to the MAC CRC). Figure 3 shows how ARQ triggered retransmissions work. In the Figure, the payload of packet $\mathrm{X}$ is corrupted to such an extent that it cannot be reconstructed. Therefore, in packet $\mathrm{X}+1$ some extra redundant data is included up to the level that decode failure is no longer practically certain. If the extra redundant data is insufficient to reconstruct the original packet's payload, the packet is simply dropped. Otherwise, of course, it is passed to the H.264/AVC decoder.

\subsection{Adaptation}

The instantaneous probability of byte loss, $B L$ (taken from a probability distribution with mean $B L_{\text {mean }}$ ) is used to calculate the amount of redundant data adaptively added to the payload. The details of how $B L$ is found are given in Section 5.2. If the original packet length is $L$, then the redundant data is given simply by

$$
\begin{aligned}
R & =L \times B L+\left(L \times B L^{2}\right)+\left(L \times B L^{3}\right) \ldots \\
& =L /(1-B L)-L,
\end{aligned}
$$


which adds successively smaller additions of redundant data, based on taking the previous amount of redundant data multiplied by $B L$.

If it turns out that the packet cannot be reconstructed, despite the provision of redundant data, then additional redundant data are added to the next packet (Extra redundant data in Fig. 3).

It is implied from Eq. (5) that if less than $k$ symbols (bytes) in the payload are successfully received then a further $k-m+e$ extra redundant bytes can be sent to reduce the risk of failure. In the evaluation tests, $e$ was set to four, resulting in a risk of failure of $8.7 \%$ (from Eq. (5)) in reconstructing the original packet if the extra redundant data successfully arrives. This reduced risk arises because of the exponential decay of the risk that is evident from Eq. (5).

\subsection{Slice duplication}

In the protection scheme, one or more of the data-partitions is duplicated so that dropped packets can be replaced. During bursts it is possible that a packet and its duplicate replacement are both affected by channel noise. Thus, extra redundant data are transmitted in an attempt to reconstruct the packet for the least affected of the two packets (the original and copy).

Rather than duplicating packets, as mentioned in Section 1, it is possible to send a reduced-quality version of a slice, which in H.264/AVC is referred to as a redundant picture slice [37]. However, employing a coarser quantization than in the main stream, can lead to drift between the encoder and decoder, as the encoder never knows which version of the slice has been decoded. Besides, replacing one partition with a redundant slice with a different QP to the other partitions would not even permit reconstruction. It should, however, be noted that in some circumstances, employing redundant picture slices is acceptable. For example, in [26] for an ad hoc network with routing path diversity, redundant picture slices were sent across one of the routes, as a guard against loss of packets in another route.

A possibility [37] is to use correctly-received reference pictures for reconstruction of redundant pictures rather than the reference pictures used by primary pictures. The decoder is able to select from a set of potential replacement redundant pictures according to the possibility of correct reconstruction. Alternatively, in [8], MBs were selected for their relative impact on reconstruction and placed within FMO slices, at some increase in computational complexity.

\section{Simulation model}

This Section discusses the simulation settings for the evaluation of the protection scheme in the following Section.

\subsection{WiMAX modeling}

To establish the behavior of rateless coding under WiMAX the well-known ns-2 simulator was augmented with a module from the Chang Gung University, Taiwan [33] that has proved an effective way of modeling IEEE 802.16e's behavior.

To evaluate the scheme, transmission over WiMAX was carefully modeled. The PHY layer settings selected for WiMAX simulation are given in Table 3. The antenna heights are typical ones taken from the standard [10]. The antenna is modeled for comparison purposes as a half-wavelength dipole, whereas a sectored set of antenna on a mast might be used in practice to achieve directivity and, hence, better performance. The IEEE 802.16 TDD frame length was set to $5 \mathrm{~ms}$, as only this value is supported in 
Table 3

IEEE 802.16e parameter settings

\begin{tabular}{ll}
\hline Parameter & Value \\
\hline PHY & OFDMA \\
Frequency band & $5 \mathrm{GHz}$ \\
Bandwidth capacity & $10 \mathrm{MHz}$ \\
Duplexing mode & TDD \\
Frame length & $5 \mathrm{~ms}$ \\
Max. packet length & $1024 \mathrm{~B}$ \\
Raw data rate & $10.67 \mathrm{Mbps}$ \\
IFFT size & 1024 \\
Modulation & $16-\mathrm{QAM} 1 / 2$ \\
Guard band ratio & $1 / 8$ \\
MS transmit power & $245 \mathrm{~mW}$ \\
BS transmit power & $20 \mathrm{~W}$ \\
Approx. range to SS & $1 \mathrm{~km}$ \\
Antenna type & Omni-directional \\
Antenna gains & $0 \mathrm{dBD}$ \\
SS antenna height & $1.2 \mathrm{~m}$ \\
BS antenna height & $30 \mathrm{~m}$ \\
\hline
\end{tabular}

OFDMA $=$ Orthogonal Frequency Division Multiple Access, QAM = Quadrature Amplitude Modulation, TDD = Time Division Duplex.

the WiMAX forum simplification of the standard [30]. The data rate results from the use of one of the mandatory coding modes [10] for a TDD downlink/uplink sub-frame ratio of 3:1. The WiMAX base station (BS) was assigned more bandwidth capacity than the uplink to allow the BS to respond to multiple mobile subscriber stations (SSs). Thus, the parameter settings in Table 3, such as the modulation type and physical-layer coding rate, are required to achieve a data-rate of $10.67 \mathrm{Mbps}$ over the downlink.

In order to introduce sources of traffic congestion, an always available FTP source was introduced with TCP transport to a second SS. Likewise, a CBR source with packet size of $1000 \mathrm{~B}$ and inter-packet gap of $0.03 \mathrm{~s}$ was also downloaded to a third SS. While the CBR and FTP traffic occupies WiMAX's non-rtPS (non-real-time polling service) queue, rather than the rtPS queue, they still contribute to packet drops in the rtPS queue for the video, if the packet rtPS buffer is already full or nearly full, while the nrtPS queue is being serviced. Sender buffer sizes were set to fifty packets (WiMAX MAC Protocol Data Units (PDUs) [3]), as is normal to reduce buffer waiting time and reduce energy consumption at the SSs. For clarity of interpretation of the results, this paper does not model horizontal handovers, though it should be noted these are a real possibility that can disrupt video streams, and in [6] there is a proposal to speed-up connection set-up in WiMAX handovers. Related work on efficient key exchange during mobile roaming can be found in [15].

\subsection{Channel model}

A two-state Gilbert-Elliott channel model [38] was used in the physical layer of the simulation to simulate the channel model for WiMAX. To model the effect of slow fading at the packet-level, the $P G G$ (probability of being in a good state) was set to $0.95, P B B$ (probability of being in a bad state) $=0.96$, $P G$ (probability of packet loss in a good state) $=0.02$ and $P B$ (probability of packet loss in a bad state) $=0.01$ for the Gilbert-Elliott parameters. Additionally, it is still possible for a packet not to be dropped in the channel but, nonetheless to be corrupted through the effect of fast fading (or other sources of noise 
and interference). This byte-level corruption was modeled by a second Gilbert-Elliott model, with the same parameters (applied at the byte level) as that of the packet-level model except that $P B$ (probability of byte loss) was increased to 0.165 . The Gilbert-Elliott scheme, though it is simple, has been widely adopted [13], as it is thought to realistically model the error bursts that are apparent to the application layer and, more significantly, can be particularly damaging to compressed video streams, because of the predictive nature of source coding. Therefore, the impact of error bursts should be assessed [20] in video streaming applications.

In the adaptive scheme, the probability of channel byte loss serves to predict the amount of redundant data to be added to the payload. If $P G B$ and $P B G$ are the probabilities of going from good to bad state and from going from bad to good state respectively, then

$$
\begin{aligned}
& \pi_{G}=P B G /(P B G+P G B) \\
& \pi_{B}=P G B /(P B G+P G B)
\end{aligned}
$$

are the steady state probabilities of being in the good and bad states. Consequently, the mean probability of channel corruption is given by

$$
B L_{\text {mean }}=P G \cdot \pi_{G}+P B \cdot \pi_{B}
$$

that is the mean of a Uniform distribution in this case.

The WiMAX standard specifies that a station should provide channel measurements that can form a basis for channel quality estimates. These are either Received Signal Strength Indicators or may be Carrier-to-Noise-and-Interference Ratio measurements made over modulated carrier preambles. In a simulation, assuming perfect channel knowledge of the channel conditions when the original packet was transmitted establishes an upper bound beyond which the performance of the adaptive scheme cannot improve. However, we have included measurement noise to test the robustness of the scheme. Measurement noise was modelled as a zero-mean Gaussian (normal) distribution and added up to a given percentage to the packet loss probability estimate.

\subsection{Video configuration}

The Football video sequence was also employed for the WiMAX downlink tests. As a GOP structure of IPPP... was employed, it is necessary to protect against error propagation in the event of inter-coded P-picture slices being lost. By default, to ensure higher quality video, 5\% intra-coded MBs (randomly placed) were included in each frame (apart for the first I-picture) to act as anchor points in the event of slice loss. The JM 14.2 version of the H.264/AVC codec software was employed, with the Evalvid framework [16] used to reconstruct sequences, according to reported packet loss patterns from the simulator, and to assess the objective video quality (PSNR) relative to the input YUV raw video. Lost partition-C slice packets were compensated for by motion-copy error concealment at the decoder using the MVs in partition-A. From Section 2, for high- to medium-quality video, the size of partition-A is relatively smaller in length than the other two partitions and, therefore, less likely to suffer from channel error. Similarly, partition-B is generally smaller than partition-C, provided that the intra-refresh MB contribution is kept to a low percentage (as herein). 


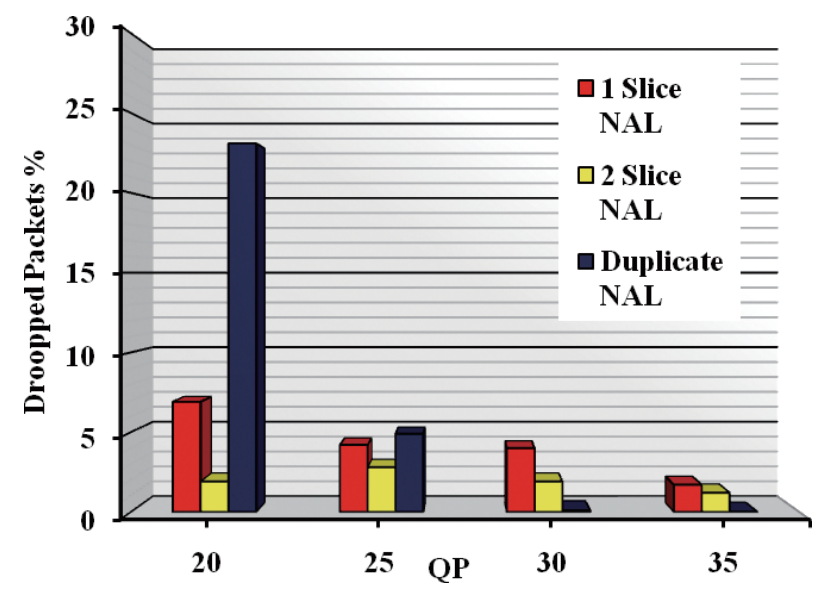

Fig. 4. Dropped packet rates when streaming Football, with different slicing configurations.

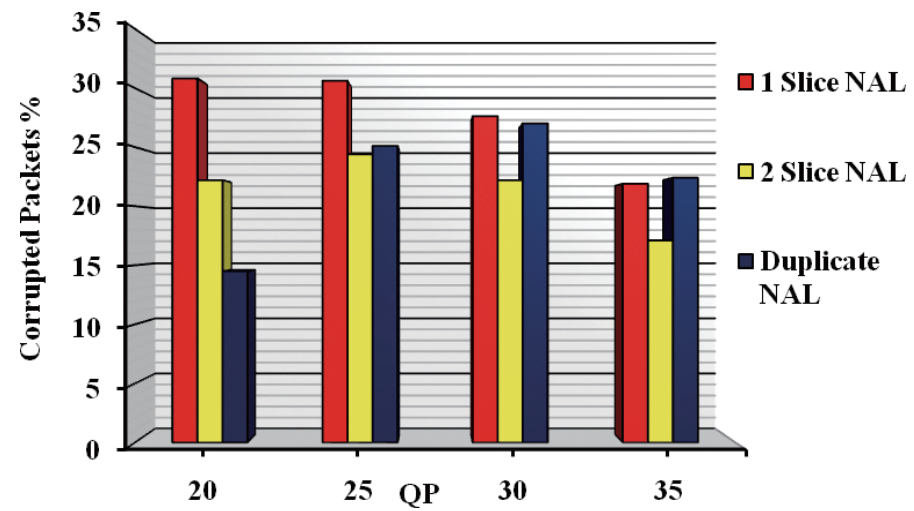

Fig. 5. Corrupted packet rates when streaming Football, with different slicing arrangements.

\section{Evaluation}

The protection scheme was evaluated for the impact of duplicate slices and the rate of intra-refresh MBs. For the former the full Gilbert-Elliott model was applied (refer to Section 5.2), while for the latter the secondary model of byte error only was applied. Three types of erroneous packets were considered: 1) packet drops at the BS sender buffer 2) packet drops through channel noise and interference, and 3) corrupted packets that were received but affected by Gilbert-Elliott channel noise to the extent that they could not be immediately reconstructed without an ARQ triggered retransmission of piggybacked redundant data. Notice that if the retransmission of additional redundant data still fails to allow the original packet to be reconstructed then the packet is simply dropped. The Raptor code Eq. (5) was applied to decide if a packet could be recovered, given the number of bytes that were declared to be in error.

\subsection{Impact of duplicate packets}

In Figs 4 to 8 , three schemes are compared, all of which adaptively include redundant data according to the description of Section 4.2. As mentioned in Section 5.2, in the mean 5\% additive Gaussian 


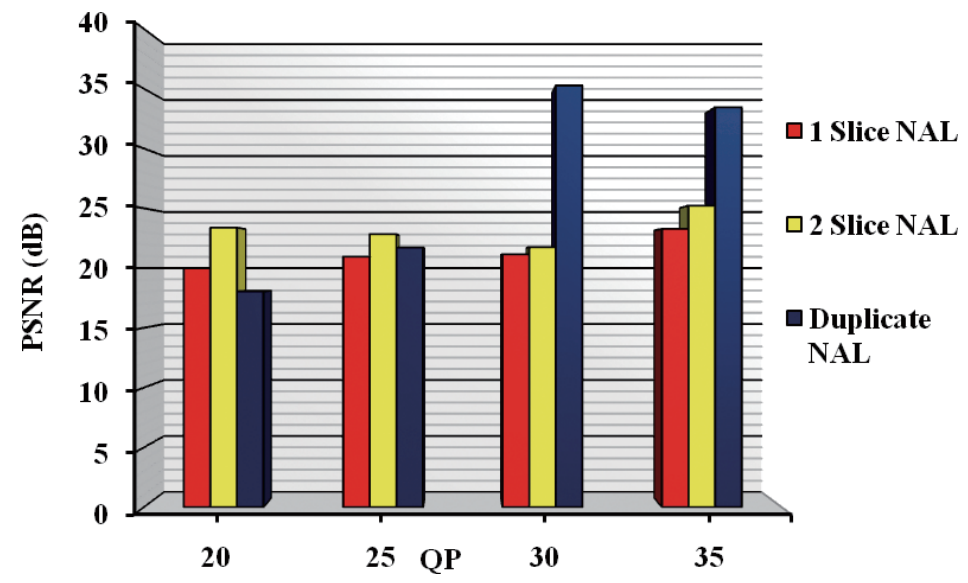

Fig. 6. Mean video quality when streaming Football, with different slicing arrangements.

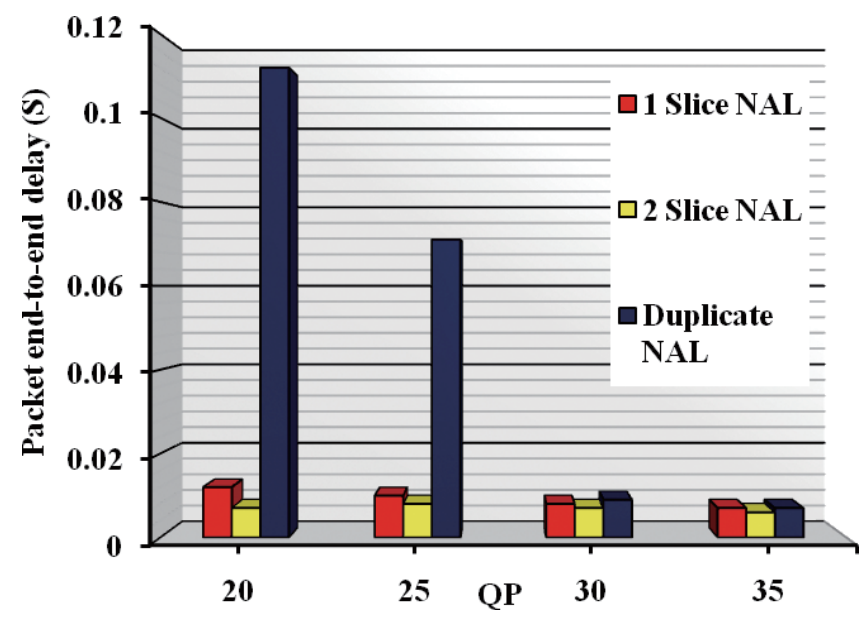

Fig. 7. Mean single transmission packet end-to-end delay when streaming Football, with different slicing arrangements.

measurement noise was applied to the estimate of $B L$ in Eq. (5) to make the adaptive schemes more realistic. The scheme labeled 1 slice $N A L$ treats a picture as a single slice but creates the three data partitions (A, B, and C) described in Section 2. The scheme labeled 2 slice NAL geometrically divides each picture into two horizontal slices. Each of the two slices is split into three data partitions and as before each forms a NAL unit. Each NAL unit occupies an IP/UDP/RTP packet, which, after packetization as a MAC Service Data Unit [3], occupies a single WiMAX MAC PDU. A number of header compression schemes are available to significantly reduce the impact of the IP/UDP/RTP overhead. The main effect of the two slice scheme is to reduce packet sizes. The scheme labeled Duplicate NAL also includes the duplicate slice packets, which in the following Figures means redundant partition-A, -B, and -C packets (all packets replicated). The Redundant NAL scheme is a single slice per picture scheme and, hence, no advantage is gained from smaller packet sizes. 5\% intra-refresh data were added to each picture, increasing the size of partition-B packets (refer to Fig. 1). In these Figures, the Duplicate NAL scheme extends to all partitions. This does not amount to a change in bitrate because the packets are simply replicated. However, the end-to-end packet delay will obviously increase because of the interleaving of 


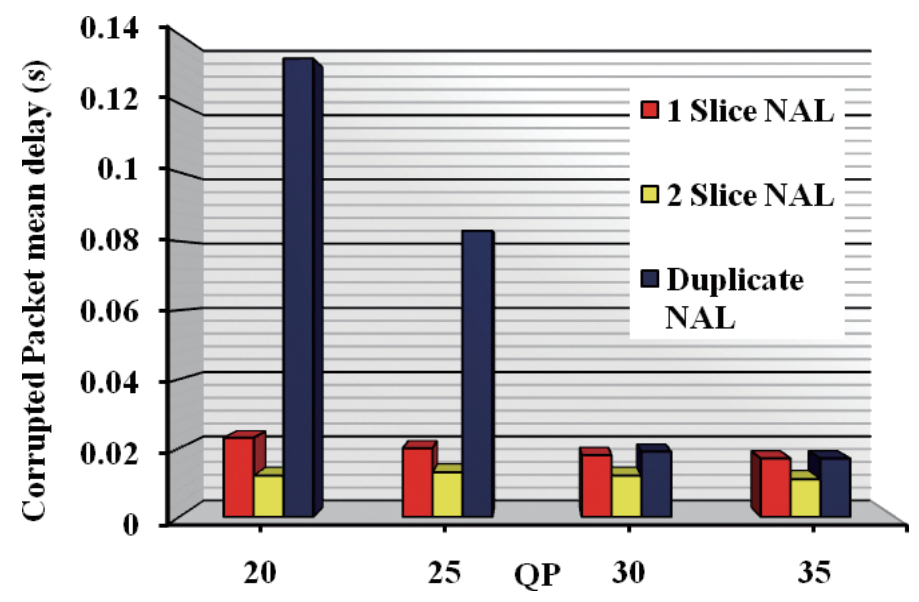

Fig. 8. Mean re-transmitted packet end-to-end delay when streaming Football, with different slicing arrangements.

the duplicate slice packets. Notice also that the number of packets sent for the two-slice scheme is the same as for the redundant slice packet scheme.

Though only a moderate percentage of one slice scheme packets $(6.9 \%)$ are dropped outright, Fig. 4, from the combined effects of congestion and channel conditions, this results at QP $=20$ to a PSNR of only $19.98 \mathrm{~dB}$, Fig. 6, that is, it is at an unacceptable level. Unfortunately, though the percentage of packets dropped reduces (because of the smaller packet sizes), the sending quality is reduced at higher QPs, with the result that the objective quality at the receiver display remains below $25 \mathrm{~dB}$, that is approximately equivalent to a poor rating in the ITU P.800's [12] mean opinion score scale. Packet size has a significant effect, as in the two-slice scheme video quality is increased. However, reducing the packet size is still insufficient (video quality below $25 \mathrm{~dB}$ ) when faced with the combined effect of packet drops and channel conditions, Fig. 5.

In the duplicate NAL scheme, retransmission of extra redundant data was scheduled for all corrupted packets, even if two packets duplicated each other. This is because it is not possible to know in advance whether the extra redundant data will arrive for any one of the two packets. This provision has a significant effect in improving the video quality at higher QPs. The reason is that retransmitting extra redundant data by two alternative means increases the chance that a packet can be reconstructed. Overall video quality is approximately equivalent (above $31 \mathrm{~dB}$ ) to the ITU P.800 'good' category at the higher QPs illustrated.

At lower QPs for the duplicate NAL scheme, higher packet drop rates occur (23.1\% and 4.1\%). The packet size at $\mathrm{QP}=20$ is large and the effect of sending such packets in duplication contributes to the high loss rate. Therefore, at low QP it appears that redundant slice provision is not an effective guard against packet drops Unfortunately also, for lower QPs total end-to-end delay for both normal, Fig. 7, and corrupted packets, Fig. 8, is high and there is a possibility of interruptions to the display, i.e. freeze frame effects. The throughput of the WiMAX channel is likely to remove the risk of display interruptions at higher QP delay times.

Figures 9 to 11 present an analysis of packet drops from packet loss alone, i.e. by application of the Gilbert-Elliot packet loss model of Section 4.2. From this analysis it is apparent that at lower QPs, packet drop numbers are distributed in inverse order of data priority. This should not be surprising as higher-priority packets (partition-A and -B packets) are smaller at lower QPs and, hence, in the mean, spend less time exposed to the channel. A surprising feature of these Figures is that in the duplicate 


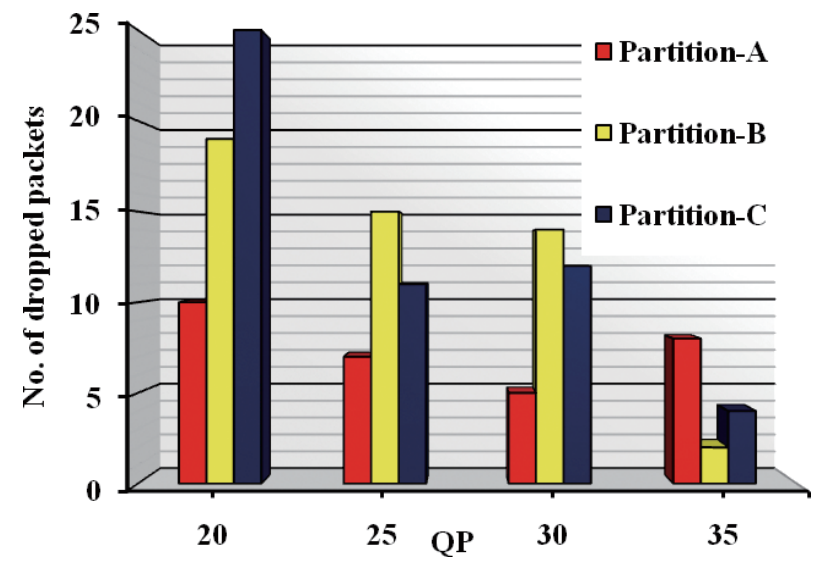

Fig. 9. Analysis of dropped packets according to partition type for one slice per picture.

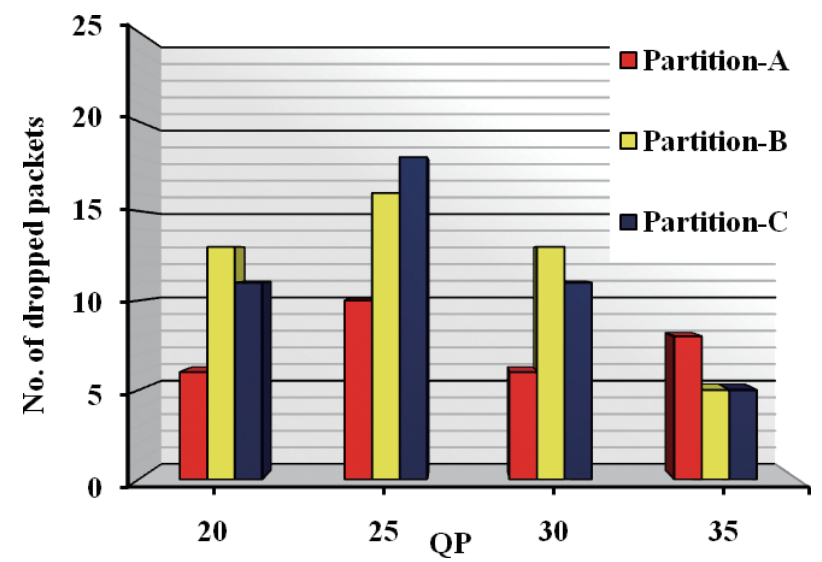

Fig. 10. Analysis of dropped packets according to partition type for two slices per picture.

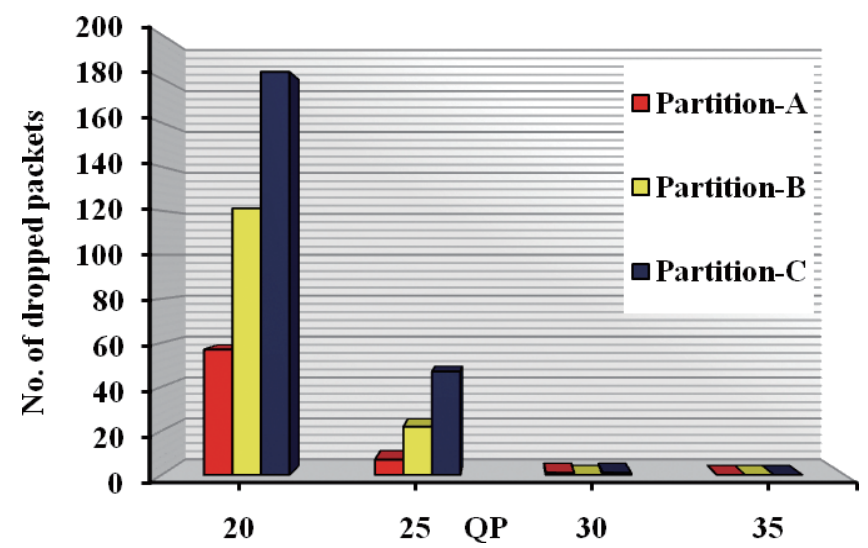

Fig. 11. Analysis of dropped packets according to partition type with duplicate slices. 


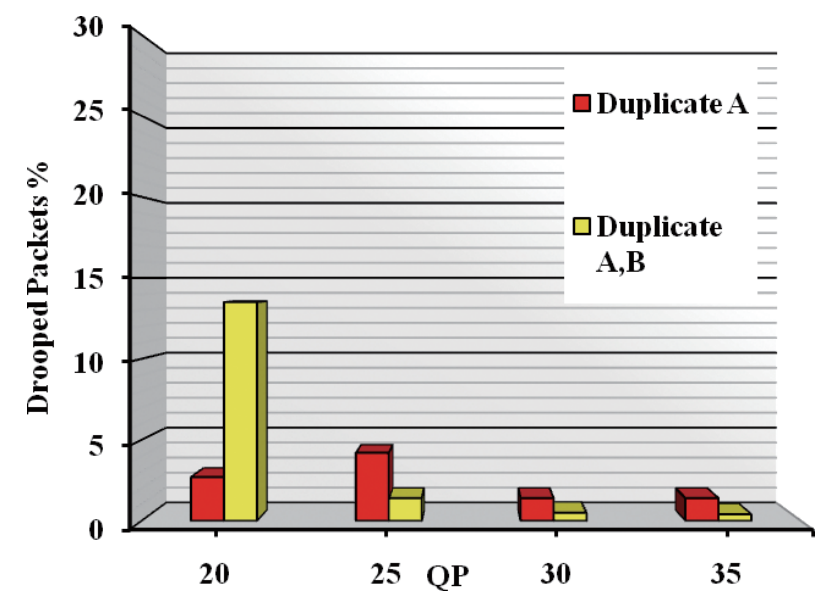

Fig. 12. Packet drop rate when streaming Football, with alternative duplicate NAL protection schemes.

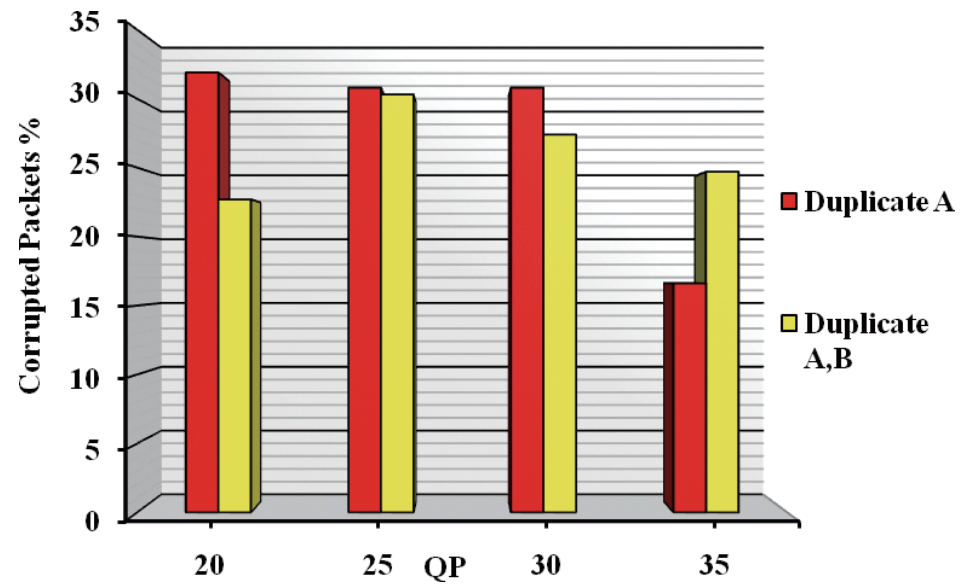

Fig. 13. Corrupted packet rate when streaming Football, with alternative duplicate NAL protection schemes.

NAL scheme at higher QPs, Fig. 11, few if any packets are dropped when traversing the channel, while at lower QPs large numbers of packets are lost through buffer overflow. Because the packet sizes are small at higher QPs, packet drops are generally less than $2 \%$ (except for $\mathrm{QP}=30$ in the one-slice NAL scheme).

Naturally it was of interest whether it was possible to employ only duplicate partition-A slice packets or only duplicate partition-A and partitition-B slice packets. These two options are examined in Figs 12 to 16. Figures 17 and 18, perform a partition-type analysis for the number of dropped packets in the selective duplication schemes and equally show that higher-priority data packets benefit from size-dependent packet losses. However, though other performance metrics are favorable, and though duplication of both partition-A and partition-B slice packets is preferable, results show that in this scenario for the Football video sequence, duplicate protection of partition-C is also necessary to reconstruct the stream to a satisfactory quality. We attribute this to the high motion present in the Football reference sequence, as this implies that succeeding frames may be very different. As a result, error concealment by motion vectors alone may be insufficient. 


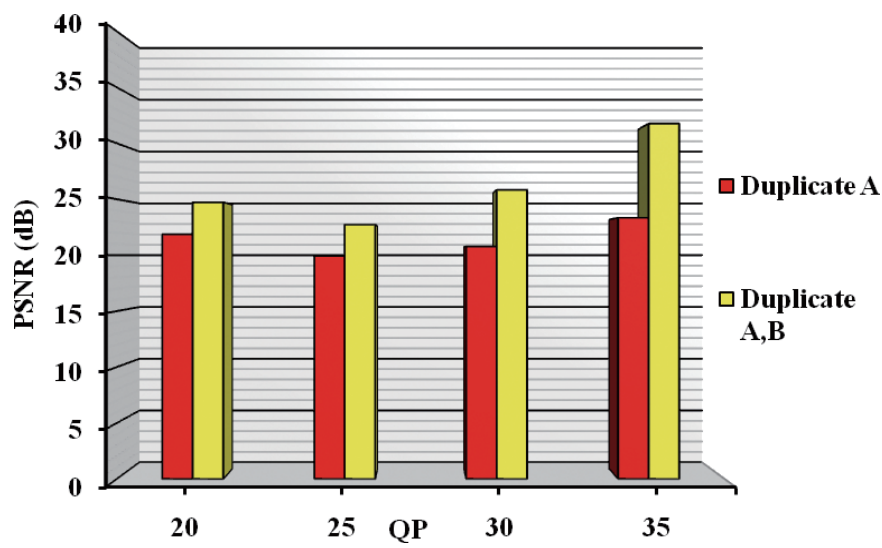

Fig. 14. Mean video quality when streaming Football, with alternative duplicate NAL protection schemes.

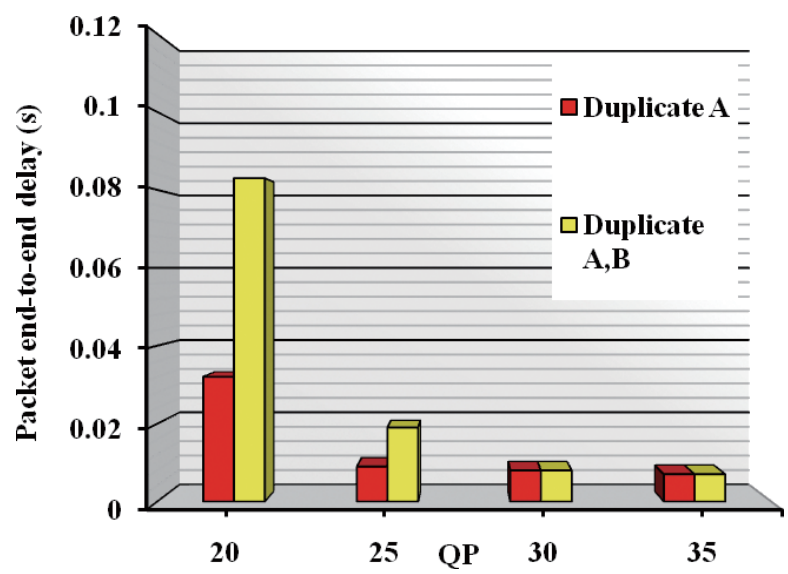

Fig. 15. Mean single transmission packet end-to-end delay when streaming Football, with alternative duplicate NAL protection schemes.

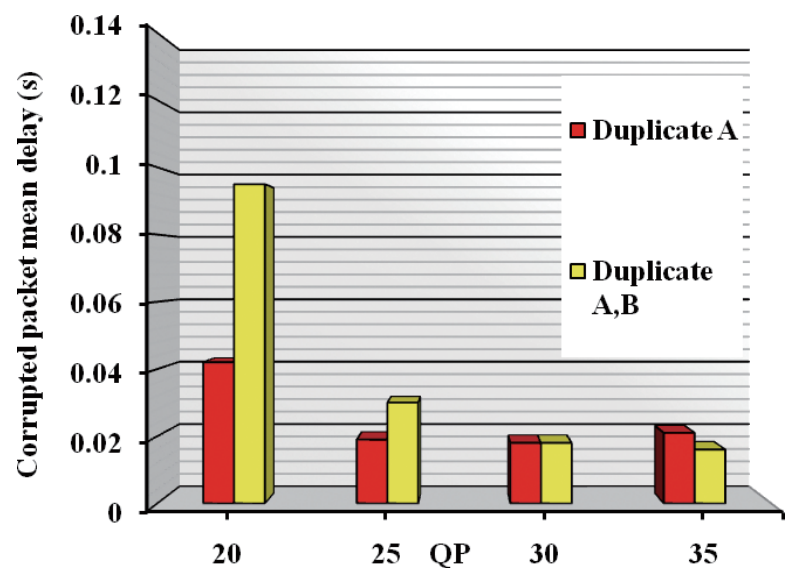

Fig. 16. Mean re-transmission packet end-to-end delay when streaming Football, with alternative duplicate NAL protection schemes. 


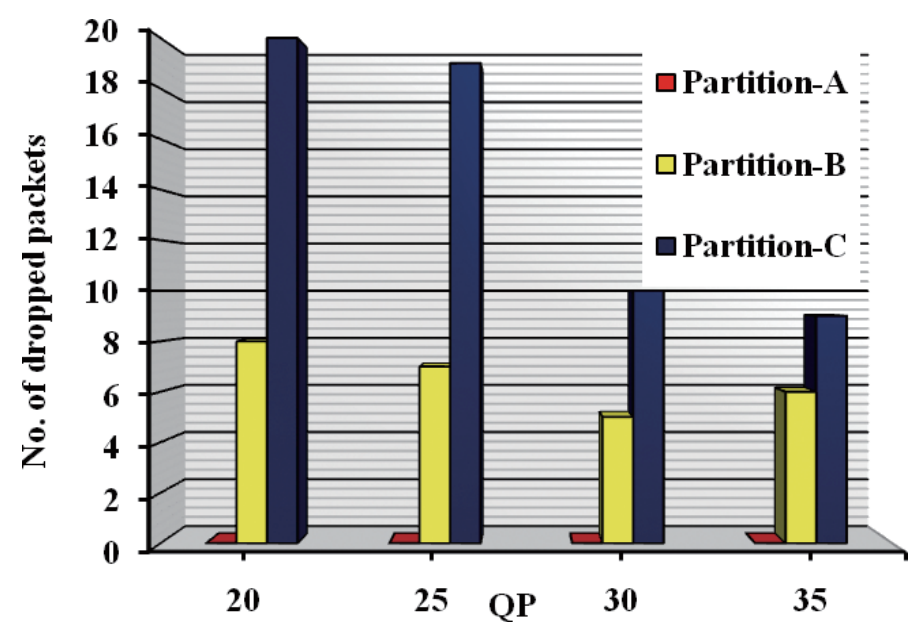

Fig. 17. Analysis of dropped packet numbers according to partition type when streaming Football, with duplicate partition-A packets.

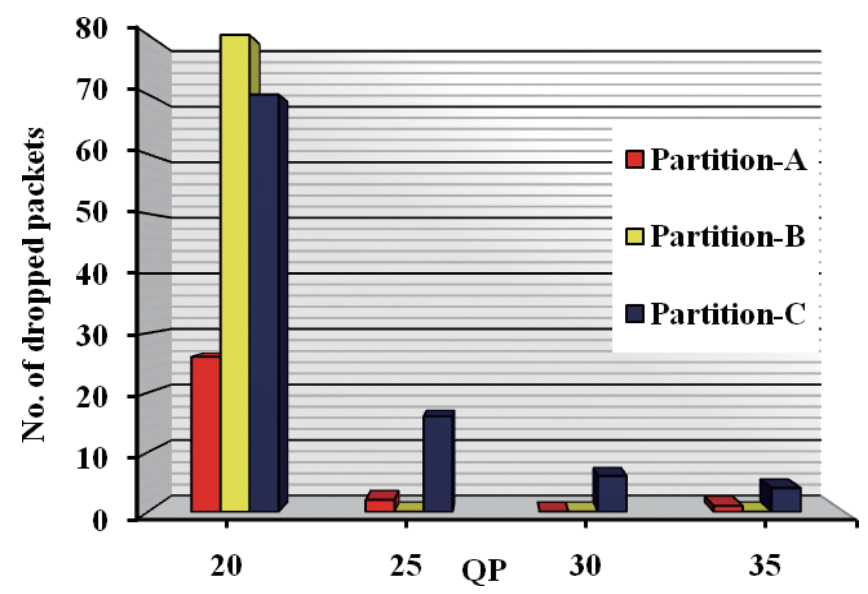

Fig. 18. Analysis of dropped packet numbers according to partition type when streaming Football, with duplicate partition-A and partition-B packets.

\subsection{Impact of intra-refresh macroblock provision}

Figures 19 to 21 show the result of retaining the 'duplicate NAL' scheme of Figs 4 to 6 but varying the provision of intra-refresh MBs. Increasing the provision of intra-refresh MB above 5\% to 6\% and higher in the case of MB line intra update, increases the throughput and, hence, the bandwidth requirements in respect to co-existing traffic. $6 \%$ rather than $5 \% \mathrm{MB}$ refresh is chosen because without naturally encoded intra-refresh MBs, then one line of MBs corresponds to about $6 \%$ of a CIF picture. From the PSNR results it can be seen that reducing the intra-refresh MB percentage to $2 \%$ actually improves the PSNR at QPs 30 and 35. The main effect of reducing the percentage of intra-refresh MBs is that the size of partition-B-bearing packets is reduced. In turn, this makes these packets less likely to be affected by channel conditions, especially burst errors arising from the simulated Gilbert-Elliott model. 


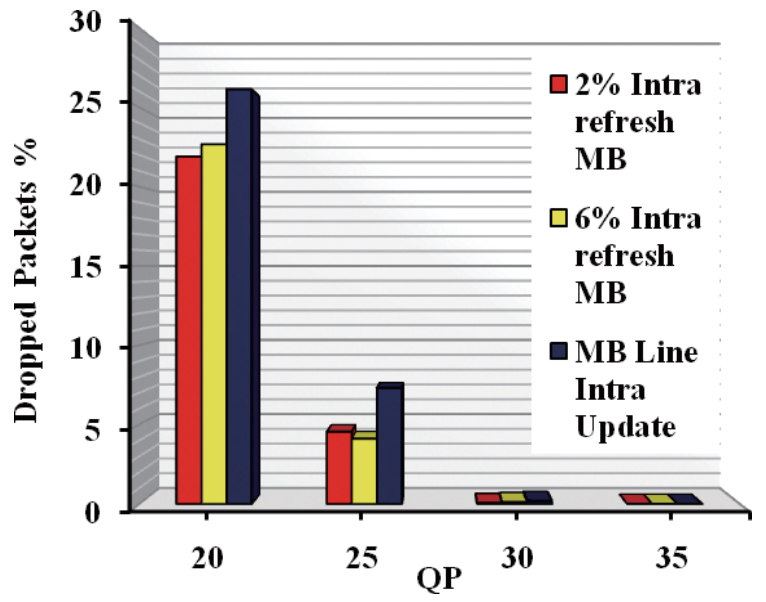

Fig. 19. Dropped packet rates when streaming Football, with duplicate NAL protection extending to all partitions and different levels of intra-refresh MB protection.

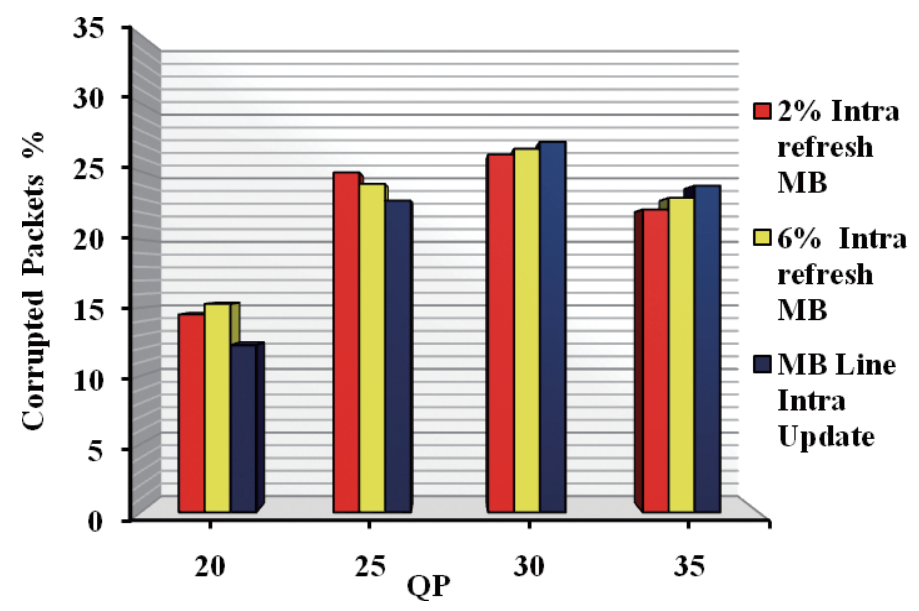

Fig. 20. Corrupted packet rates when streaming Football, with duplicate NAL protection extending to all partitions and different levels of intra-refresh MB protection.

\section{Conclusion}

Though a form of hybrid ARQ has been applied in this paper and though an advanced form of packetization (data-partitioning) was combined with adaptive rateless coding, it is still very possible that unacceptable video quality can occur when streaming over a broadband wireless channel. This is particularly so if there is high motion in the streamed video, which unfortunately includes many sports sequences. Therefore, duplication of the stream seems very necessary in harsh channel conditions, though if the channel estimation indicates that conditions are benign, then the duplicate stream can simply be turned off. The careful choice of quantization parameter for VBR video is necessary, as too low a choice will result in large packets that are prone to loss or corruption in one of several ways. A low level of intra-refresh provision is adequate and the low level results in reduced risk of packet loss. The adaptive scheme presented can be turned around in that instead of repairing lower priority data packets, these packets can be discarded if channel conditions do not merit retransmission. 


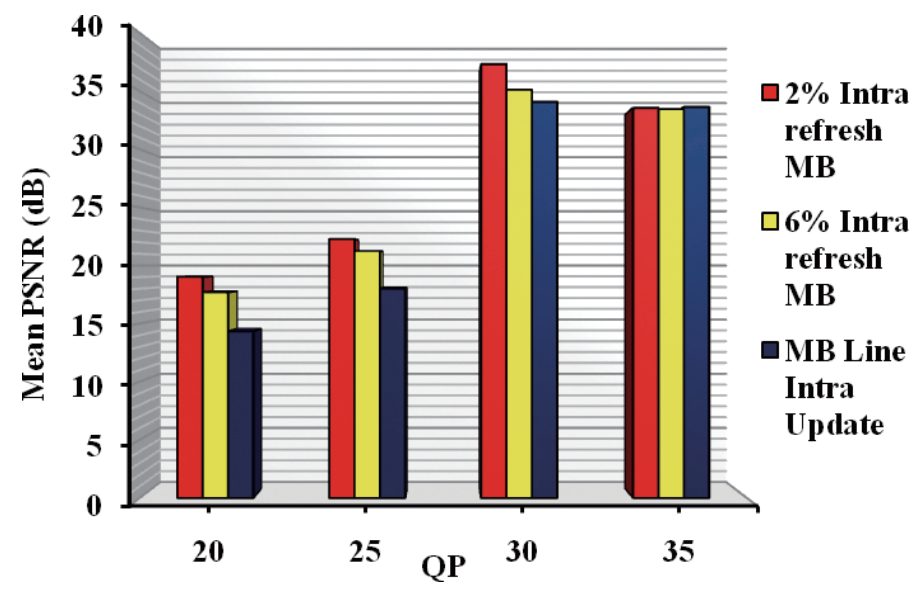

Fig. 21. Mean video quality when streaming Football, with duplicate NAL protection extending to all partitions and different levels of intra-refresh MB protection.

\section{References}

[1] F. Agboma and A. Liotta, Addressing user expectations in mobile content delivery, Mobile Information Systems 3(3/4) (2007), 153-164.

[2] S. Ahmad, R. Hamzaoui and M. Al-Akaidi, Robust live unicast video streaming with rateless codes, Proc. Int'l PacketVideo Workshop, 2007, pp. 87-84.

[3] J.G. Andrews, A. Ghosh and R. Muhamed, Fundamentals of WiMAX: Understanding broadband wireless networking, Prentice Hall, Upper Saddle River, NJ, 2007.

[4] B. Barmada, M.M. Mahdi, E.V. Jones and M. Ghanbari, Prioritiised transmission of data-partitioned H.264 video with hierarchical QAM, IEEE Sig Proc Letter 12(8) (2005), 577-580.

[5] J. Casampere, P. Sanshez, T. Villameriel and J. Del Ser, Performance evaluation of H.264/MPEG-4 Scalable Video Coding over IEEE 802.16e networks, Proc. IEEE Int'l Symp. on Broadband Multimedia Systems and Broadcasting, 2009, pp. 1-6.

[6] Y.-F. Ciou, F.-Y. Leu, Y.-L. Huang and K. Yim, A handover security mechanism employing the Diffie-Hellman key exchange approach for the IEEE 802.16e wireless networks, Mobile Information Systems 7(3) (2011), 241-269.

[7] N. Degrande, K. Laevens and D. De Vleeschauwer, Increasing the user perceived quality for IPTV services, IEEE Communs Mag 46(2) (2008), 94-100.

[8] P. Ferré, D. Agrafiotis and D. Bull, A video error resilience redundant slices algorithm and its performance relative to other fixed redundancy schemes, Image Communication 25(3) (2010), 163-178.

[9] O. Hillested, A. Perkis, V. Genc, S. Murphy and J. Murphy, Adaptive H.264/MPEG-4 SVC video over IEEE 802.16 broadband wireless access networks, Proc. Packet Video, 2007, pp. 26-35.

[10] IEEE 802.16e-2005, IEEE Standard for Local and Metropolitan Area Networks, Part 16: Air Interface for Fixed and mobile Broadband Wireless Access Systems, 2005.

[11] O. Issa, W. Li and H. Liu, Performance evaluation of TV over broadband wireless access networks, IEEE Trans Broadcasting 56(2) (2010), 201-210.

[12] ITU-T Rec. P.800, Methods for subjective testing of video quality, 1996.

[13] Ch. Jiao, L. Schwiebert and B. Xu, On modeling the packet error statistics in bursty channels, IEEE Conf on Local Computer Networks, 2002, pp. 534-541.

[14] H.-H. Juan, H.-C. Huang, C.Y. Huang and T. Chiang, Cross-layer mobile wireless MAC designs for the H.264/AVC scalable video coding, Wireless Networks 16(1) (2008), 113-123.

[15] H.G. Kim and J.H. Lee, Diffie-Hellman key based authentication in proxy mobile IPv6, Mobile Information Systems 6(1) (2010), 107-121.

[16] J. Klaue, B. Rathke and A. Wolisz, EvalVid - A framework for video transmission and quality evaluation, Proc. Int'l Conf. on Modeling Techniques and Tools for Computer Performance, 2003, pp. 255-272.

[17] E. Krause et al., Method and apparatus for refreshing motion compensated sequential video images, US 5,057,916, United States Patent Office, 1991.

[18] L.M. Lee, H.-J. Park, S.G. Choi and J.K. Choi, Adaptive hybrid transmission mechanism for on-demand mobile IPTV over WiMAX, IEEE Trans Broadcasting 55(2) (2009), 468-477. 
[19] A.H. Li, S. Kittitornkun, Y.H. Hu, D.S. Park and J.D. Villasenor, Data partitioning and reversible variable length codes for robust video communications, Proc. of Data Compression Conf., 2002, pp. 460-489.

[20] Y.-J. Liang, J.G. Apostolopoulos and B. Girod, Analysis of packet loss for compressed video: Effect of burst losses and correlation between error frames, IEEE Trans Circ Systems Video Technol 18(7) (2008), 861-874.

[21] Y.-J. Liang, K. El-Maleh and S. Manjunath, Upfront intra-refresh decision for low-complexity wireless video telephony, IEEE Int Symposium on Circuits and Systems (2006).

[22] M. Luby, T. Gasiba, T. Stockhammer and M. Watson, Reliable multimedia download delivery in cellular broadcast networks, IEEE Trans Broadcasting 53(1) (2007), 235-246.

[23] D.J.C.MacKay, Fountain codes, IEE Proc.: Communications 152(6) (2005), 1062-1068.

[24] S. Mys, P. Lambert and W. De Neve, SNR scalability in H.264/AVC using data partitioning, Proc. Pacific Rim Conf. in Multimedia, 2006, pp. 329-338.

[25] R. Palanki and J. Yedidai, Rateless codes on noisy channels., Proc. Int'l Symp Inform Theory 2004, p. 37.

[26] N. Qadri, M. Altaf, M. Fleury, and M. Ghanbari, Mobile Information Systems 6(3) (2010), 259-280.

[27] I. Radulovic, Y.-K. Wang, S. Wenger, A. Hallapuro, M.H. Hannuksela and P. Frossard, Multiple description H.264 video coding with redundant pictures, Proc. Int'l Workshop on Mobile Video, 2007, pp. 37-42.

[28] R.M. Schreier and A. Rothermel, Motion adaptive intra refresh for the H.264 video coding standard, IEEE Trans Consumer Electronics 52(1) (2006), 249-253.

[29] A. Shokorallahi, Raptor codes, IEEE Trans Information Theory 52(6) (2006), 2551-2567.

[30] C. So-In, R. Jain and A.-K. Tamini, Capacity evaluation of IEEE 802.16e WiMAX, J. of Computer Systems, Networks, and Communications, [online], 2010, p. 12.

[31] A.M. Tourapis, K. Sühring and G. Sullivan, H.264/14496-10 AVC Reference Software Manual, 31st Meeting of the Joint Video Team, London, 2009.

[32] T.D. Tran, L.-K. Liu and P.H. Westering, Low-delay MPEG-2 video coding, Proc SPIE - Int Soc Opt Eng (USA) 3309 (1997), 510-516.

[33] F.C.D. Tsai et al., The design and implementation of WiMAX module for ns-2 simulator, Workshop on ns2, article no. 5, 2006.

[34] D. de Vleeschauwer and K. Laevens, Performance of caching systems for IPTV on-demand services, IEEE Trans Broadcasting 55(2) (2009), 491-501.

[35] S. Wenger, H.264/AVC over IP, IEEE Trans Circuits Syst Video Technol 13(7) (2003), 645-655.

[36] M. Wu, S. Makharia, H. Liu, D. Li and S. Mathur, IPTV multicast over wireless LAN using merged hybrid ARQ with staggered adaptive FEC, IEEE Trans Broadcasting 55(2) (2009), 363-374.

[37] C. Zhu, Y.K. Wang, M. Hannuksela and H. Li, Error resilient video coding using redundant pictures, Proc IEEE Int'l Conf on Image Processing, 2006, pp. 801-804.

[38] M. Zorzi and R. Rao, On the statistics of block errors in bursty channels, IEEE Trans Commun 45(6) (1997), 660-667.

Laith Al-Jobouri received his BSc in 1995 from the University of Technology, Iraq and subsequently worked in different wireless and microwave projects. He obtained his MSc from the University of Mosul, Iraq in 2001 and then again returned to work as a project manager and head of department for several national and international companies concerned with wireless and GSM. He is now undertaking PhD research for a degree at the University of Essex, UK. His research interests are in video over wireless, forward error correction, and wireless technologies.

Martin Fleury holds a degree in Modern History (Oxford University, UK) and a Maths/Physics based degree from the Open University, Milton Keynes, UK. He obtained an MSc in Astrophysics from QMW College, University of London, UK in 1990 and an MSc from the University of South-West England, Bristol in Parallel Computing Systems in 1991. He holds a PhD in Parallel Image-Processing Systems from the University of Essex, Colchester, UK. He worked as a Senior Lecturer at the University of Essex, where he is still a Visiting Fellow. He is now a free-lance consultant. Martin has authored or co-authored two hundred and thirty articles and book chapters on topics such as document and image compression algorithms, performance prediction of parallel systems, software engineering, reconfigurable hardware and vision systems. His current research interests are video communication over wireless networks. He has published a book on high-performance computing for low-level image- and signal-processing algorithms and a number of book chapters and has another book in print on peer-to-peer video streaming.

Mohammed Ghanbari is best known for his pioneering work on two-layer video coding for ATM networks (which earned him an IEEE Fellowship in 2001), now known as SNR scalability in the standard video codecs. He has served as an Associate Editor to IEEE Trans. on Multimedia (IEEE-T-MM from 1998-2004) He has registered for eleven international patents on various aspects of video networking and was the co-recipient of A.H. Reeves prize for the best paper published in the 1995 Proc. of IEE on the theme of digital coding. He is the co-author of "Principles of Performance Engineering", a book published by IET press 
in 1997, the author of "Video Coding: An Introduction to Standard Codecs", a book also published by IET press in 1999, which received the year 2000 best book award by the IEE, and the author of "Standard Codecs: Image Compression to Advanced Video Coding" also published by the IET press in 2003. Prof. Ghanbari has authored or co-authored at least 500 journal and conference papers, many of which have had a fundamental influence in this field. 

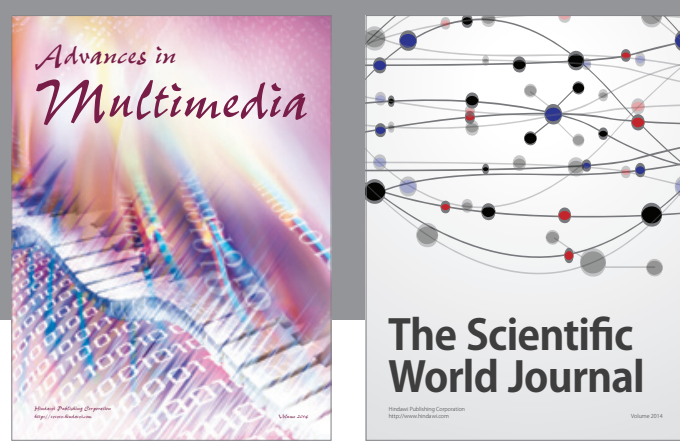

The Scientific World Journal
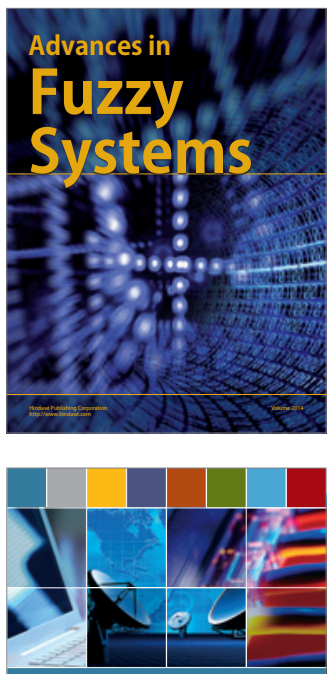

Computer Networks and Communications
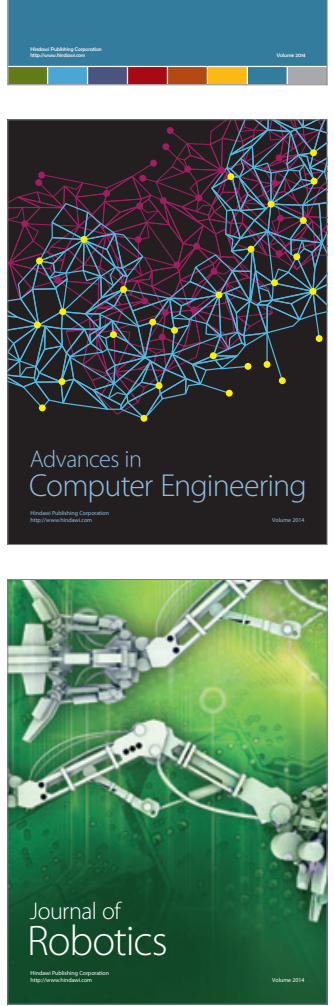
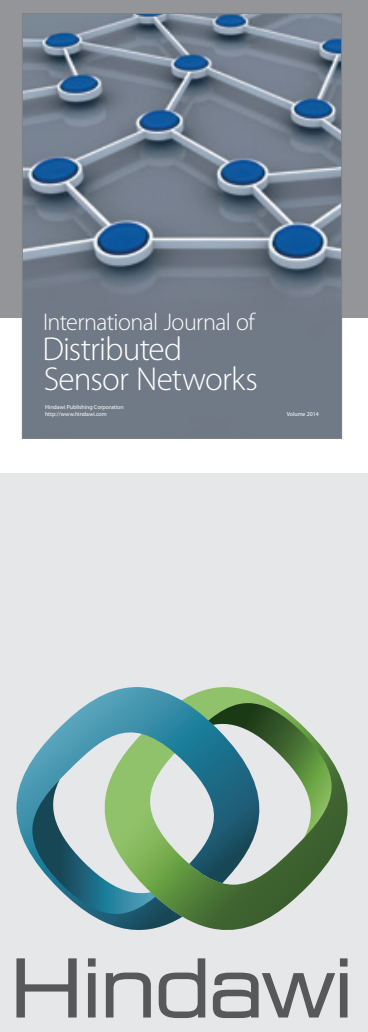

Submit your manuscripts at

http://www.hindawi.com
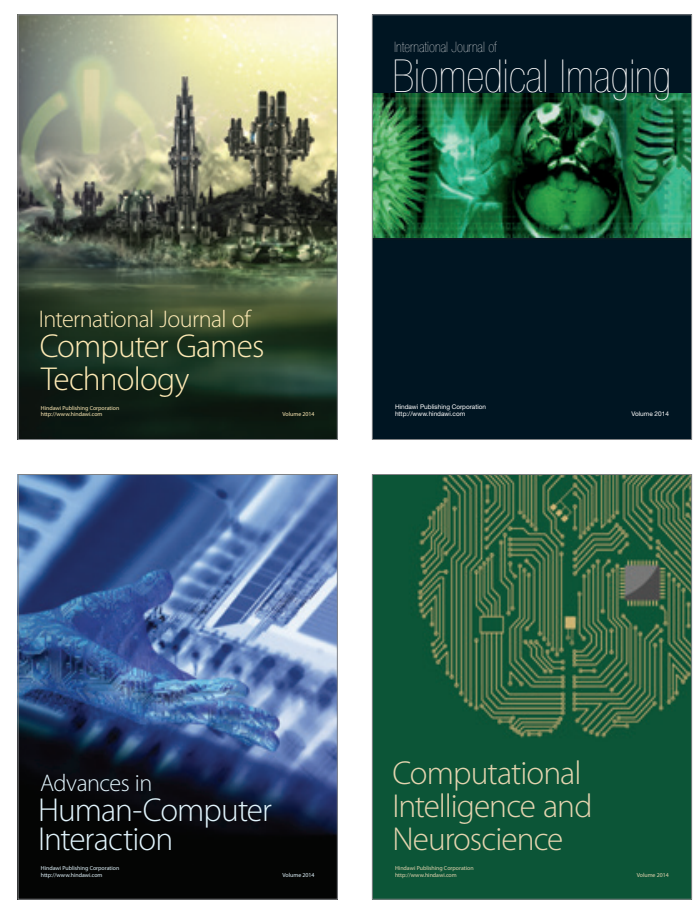
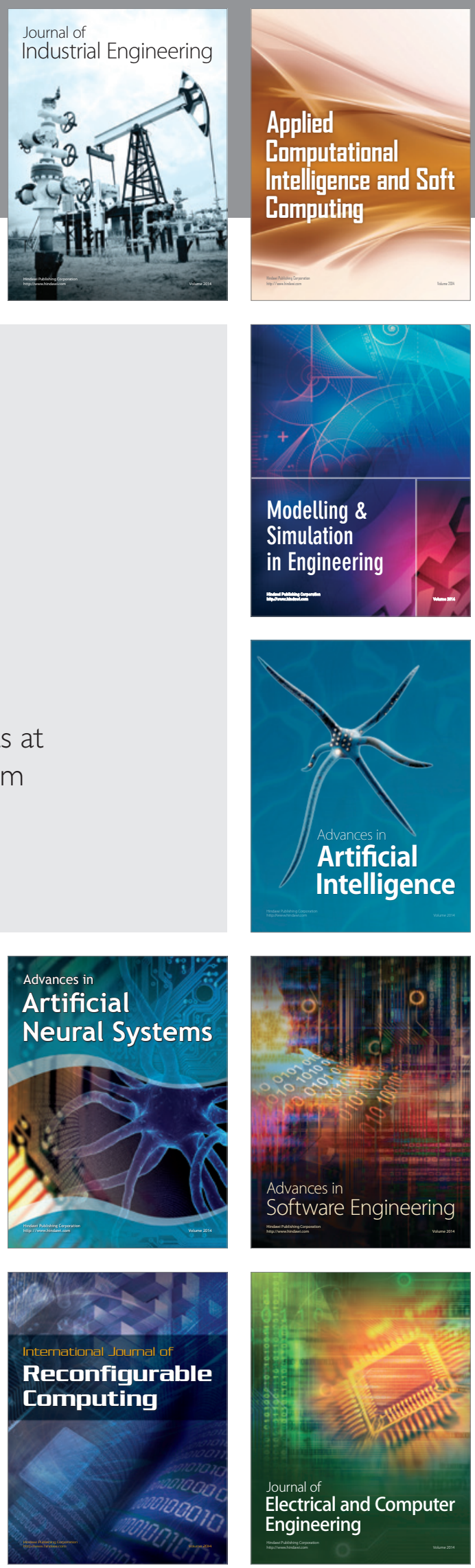